\title{
Rational Construction of Superhydrophobic PDMS/PTW@cotton Fabric for Efficient UV/NIR Light Shielding
}

Jianying Huang ( $\sim$ jyhuang@fzu.edu.cn )

Fuzhou University https://orcid.org/0000-0003-0515-9523

\section{Gang Shen}

Fuzhou University

Yimeng Ni

Fuzhou University

Kim Hoong Ng

Ming Chi University of Technology

Tianxue Zhu

Fuzhou University

Shuhui Li

Fuzhou University

Xiao Li

Fuzhou University

Weilong Cai

Fuzhou University

\section{Zhong Chen}

Nanyang Technological University

\section{Research Article}

Keywords: Passive daytime radiative cooling, Self-cleaning, Superhydrophobic, Dip coating, Cotton fabric

Posted Date: September 27th, 2021

DOl: https://doi.org/10.21203/rs.3.rs-906978/v1

License: (c) (i) This work is licensed under a Creative Commons Attribution 4.0 International License.

Read Full License 


\title{
Rational construction of superhydrophobic PDMS/PTW@cotton fabric for efficient UV/NIR light shielding
}

\author{
Jianying Huang ${ }^{*}, \mathrm{a}, \mathrm{b}$, Gang Shen ${ }^{\mathrm{a}}$, Yimeng $\mathrm{Ni}^{\mathrm{a}}$, Kim Hoong $\mathrm{Ng}^{\mathrm{c}}$, Tianxue Zhu ${ }^{\mathrm{a}}$, \\ Shuhui Li ${ }^{\mathrm{a}}$, Xiao Li ${ }^{\mathrm{a}, \mathrm{b}}$, Weilong Cai ${ }^{\mathrm{a}, \mathrm{b}}$, Zhong Chen ${ }^{\mathrm{d}}$

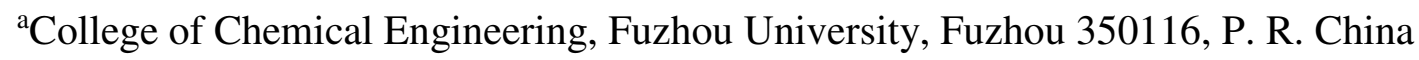

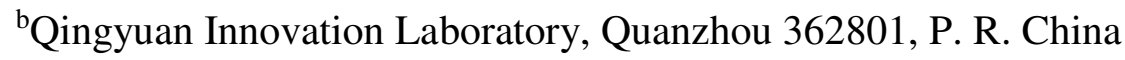 \\ ${ }^{\mathrm{c}}$ Department of Chemical Engineering, Ming Chi University of Technology, New \\ Taipei City 24301, Taiwan \\ ${ }^{\mathrm{d} S c h o o l}$ of Materials Science and Engineering, Nanyang Technological University, 50 \\ Nanyang Avenue, Singapore \\ Corresponding author email: jyhuang@fzu.edu.cn
}




\section{Abstract}

Passive daytime radiative cooling (PDRC) material has intrigued increasing attentions with its energy saving potential and smart cloth feature. In this work, PDRC cotton fabric with superhydrophobicity, ultraviolet protection and self-cleaning competency was successful constructed through the deposition chemically-stable potassium titanate whiskers (PTW) and polydimethylsiloxane (PDMS) onto cotton fibers. While featured with ultra-high contact angle of $151.9 \pm 0.9^{\circ}$, the synthesized fabric marked an average temperature drop of $\sim 5.1^{\circ} \mathrm{C}$, bestowed to its high sunlight reflectivity of $83 \%$ and infrared emissivity of nearly $90 \%$. On the other hand, real human tests further confirmed the practicality of the modified cotton fabric, with the recorded temperature drops ranged from $3.1 \sim 4.7{ }^{\circ} \mathrm{C}$ under direct sunlight. Such performance elucidated a significant improvement upon PTW/PDMS modification, which outperformed that of pristine cotton fabric. Surmising from these, the synthesized superhydrophobic fabric exhibits advantageous techno-economical index with its excellent performance and simple preparation, therefore manifesting limitless application potential, particularly in outdoor clothing and other facilities.

Keywords: Passive daytime radiative cooling; Self-cleaning; Superhydrophobic; Dip coating; Cotton fabric 


\section{Introduction}

Excessive outdoor heat stress could cast serious public health threat and curtail industrial productivity, thereby impacting the wellness and economy of the entire society. Commonly, areas with tropical climates are exposed to high temperatures throughout the year and have unbearable heat waves. Such high ambient temperature could perturb the inherent regulating mechanism of human while exposing us to the risks of life-endangering heat strokes from the excessive heat accumulation (Barros SC and Silva MM, 2018; Cai LL et al. 2018; Spector JT et al. 2016). Meanwhile, past analysis also indicated shortening of service life for outdoor products under stringent environment (Kjellstrom T et al. 2016). Machinery cooling, such as air conditioners and fans, is indispensable to alleviate the heat in summer, but only subjected to the closedenvironment in a considerable energy consumption. While similar cooling strategy is not applicable for open area, effective measure could be attained through the innovation of smart clothing with thermal radiation control (Cai LL et al. 2018; Lian YL et al. 2020; Miao DG et al. 2017; Panwar K et al. 2017; Peng LH et al. 2019a; Song YN et al. 2018; Sun KY et al. 2021; Yang YX et al. 2021; Yu X et al. 2019). Unfortunately, traditional textiles have limited performance in this respect, therefore urging a revolutionary modification of the present textile technologies to address such shortcoming.

Significantly, PDRC can be considered as an effective strategy to effectuation outdoor daytime cooling (Lu Y et al. 2019; Peng LH et al. 2019b; Wong A et al. 2015; Yang M et al. 2020; Yuan H et al. 2020; Zhou Y et al. 2019). Such process potentiates electricity-free cooling by reflecting solar spectrum, with wavelength ranged from 0.4- 
$2.5 \mu \mathrm{m}$, while emitting heat through the window of the atmosphere into the cold space (wavelengths $\sim 8-13 \mu \mathrm{m}$ ). To date, innumerable approaches have been employed for the synthesis of PDRC materials, with nano-doping (Hsu PC et al. 2016; Wu K et al. 2019) and photonic structuring (Catrysse PB et al. 2016; Fan WJ et al. 2020; Raman AP et al. 2014; Zhang HW et al. 2020) being widely investigated. For example, Zhai et al (Zhai $\mathrm{Y}$ et al. 2017) embedded silicon dioxide $\left(\mathrm{SiO}_{2}\right)$ microspheres as the emissive layer in the transparent polymer, which supplemented with an additional silver coating as the reflective layer. Such metamaterial permitted an excellent noon-time radiative cooling power of $93 \mathrm{~W} / \mathrm{m}^{2}$ under direct sunlight. On the other hand, Qi et al (Qi YL et al. 2017) proposed a composite cooling material based on $\mathrm{TiO}_{2}$-modification of hydrophobic acrylonitrile-styreneacrylate terpolymer. Significantly, a maximum temperature reduction of nearly $27^{\circ} \mathrm{C}$ (from 28 to $30^{\circ} \mathrm{C}$ ) was observed as the synthesized material was assessed in indoor, while its outdoor performance conferred a considerable cooling effect too, judging from the temperature gradient of $9{ }^{\circ} \mathrm{C}$ achieved (from 28 to $42{ }^{\circ} \mathrm{C}$ ). In addition, by replicating the unique properties of desert ants and chameleons (Shi NN et al. 2015), a scalable selective emitter based on corrugated nickel has been proposed to provide dynamic thermal control for the attainment of adjustable thermal emittance (Sala-Casanovas M et al. 2019). However, this method is exorbitant to be applied in human clothing, attributed to the complex structural designs that requiring fine nanostructuring machinery. Meanwhile, Huang et al (Huang WL et al. 2021) reported a simple "bottom-up" ball milling method for uniform micro-assembly of polyvinylidene fluoride (PVDF)-hexafluoropropene nanoparticles that rendered with high solar 
reflectivity and emissivity of $94 \%$ and $97 \%$, respectively. This method reduces the amount of volatile organic compounds used, and the coating has excellent stability, water resistance and anti-aging ability, and has great potential in practical applications.

Presently, much of the PDRC materials were mainly applied to thermal-emissive roof or paint for building (Anand J et al. 2021; Dong SM et al. 2020; Gu B et al. 2020; Huang X et al. 2020; Raman AP et al. 2014; Sabzi D et al. 2015) with the potential application in wearable fabric being overlooked. In fact, fabrics modification through incorporation of NIR reflective materials could be an effective mean to prompt PDRC effects. For example, Wong et al (Wong A et al. 2015) coated $\mathrm{TiO}_{2}$ onto cotton fabric for improved NIR reflectance, thereby yielding to better cooling effects with an additional $3.9{ }^{\circ} \mathrm{C}$ temperature reduction, as compared to that of unmodified cotton fabric. However, the binding force of PDRC of this method is usually poor. In another study, Cai et al (Cai LL et al. 2018) fabricated a $\mathrm{ZnO}-\mathrm{PE}$ nanocomposite fabric that benefiting passive outdoor cooling with its selective spectral radiating feature. By selectively radiating human heat on top of its $90 \%$ of solar reflectance, its PDRC was justified with temperature reduction of $5-13{ }^{\circ} \mathrm{C}$ under direct solar irradiation. Similarly, Song et al (Song YN et al. 2020; Song YN et al. 2018) fabricated the functionalized personal cooling textile through electrospinning of $\mathrm{PE}$, alongside with polyethylene oxide, onto a fabric. Such textile can reduce the temperature of human body by $6.8^{\circ} \mathrm{C}$ under the sunlight via $90.97 \%$ emittance of IR in wavelength of $8-13 \mu \mathrm{m}$ and $93.77 \%$ of sunlight reflectivity. However, in practice, these methods are not applicable to common fabrics, such as cotton, due to the complexity of the process. In contrast to this, 
we propose a facile dipping method herein, to meet these stringent demands of high thermal emission, selectively in the wavelength of $8-13 \mu \mathrm{m}$, as well as the strong reflection against spectrum in Vis-NIR region. Specifically, PTW was employed as PDRC material, and be deposited in the cotton fiber upon coated with PDMS binder. Meanwhile, the superhydrophobicity and self-cleaning ability were concurrently instilled into the cotton fabric, featured from the low surface energy of PDMS/PTW coating with rough surface, for greater commercial value. Results indicate that PDMS/PTW-coated samples are prevailed with higher infrared reflectivity (83\%) and emissivity (90\%), thereby exhibiting an extra temperature drop of $5.1{ }^{\circ} \mathrm{C}$ in contrast to bare cotton under direct sunlight. Meanwhile, real human tests also implied considerable cooling effects, with sensible temperature reduction of $3.1{ }^{\circ} \mathrm{C}$ to $4.7^{\circ} \mathrm{C}$ recorded under direct sunlight. Additionally, large contact angle of over $151^{\circ}$ further confirmed the success endowment of superhydrophobicity, and hence self-cleaning capability, into the fabric sample. Summarized from these, the superhydrophobic fabric is conferred to robust cooling performance in spite of its facile production, thereby manifesting great commercial value for fabrication of heat-emissive clothing and other products for application under hot sun.

\section{Experimental section}

Materials: Polydimethylsiloxane (PDMS, Sylgard-184, the ratio to curing agent is 10:1) were purchased from Dow Corning, America. Potassium titanate whiskers $\left(\mathrm{K}_{2} \mathrm{Ti}_{6} \mathrm{O}_{13}\right.$, PTW, > 99.0 \%, Shanghai Haoxi Nano Co., Ltd.). Methylene blue, acetone, ethanol was purchased from Sinopharm Chemical Reagent (China). The cotton fabric was 
purchased from Shanghai Textile Industry Institute of Technical Supervision and cut into $5 \mathrm{~cm} \times 5 \mathrm{~cm}$ before ultrasonically-cleaned by ethanol and distilled water in sequence before use.

Preparation of superhydrophobic PDMS/PTW@cotton fabric: Accuratelyweighed PDMS was pretreated by oxygen plasma before dispersing in water, followed by ultrasonication at $30-40{ }^{\circ} \mathrm{C}$ for $30 \mathrm{~min}$ to obtain homogenized suspension. Subsequently, a pre-determined proportion of PTW was slowly added into the suspension under secondary sonication of $30 \mathrm{~min}$ for better uniformity.

As shown in Scheme 1a, cotton fabric was firstly ultrasonicated in toluene solution and then ultrasonicated ethanol solution to remove the surface wax layer while exposing the hydroxyl groups of fiber to the surface. The resultant wax-removed cotton fabric was cut into $5 \mathrm{~cm} \times 5 \mathrm{~cm}$ and dipped into the as-prepared PTW/PDMS-mixed solution for $5 \mathrm{~min}$. A final drying step at $80{ }^{\circ} \mathrm{C}$ was performed on the fabric for $1 \mathrm{~h}$ to solidify PDMS/PTW microparticles.

Characterization: The morphology of the cotton samples was observed under a Hitachi S-4800 field emission scanning electron microscope (FESEM). The hydrophobicity of synthesized fabric samples was assessed by contact angle analysis that provided by Dataphysics OCA25, Germany. $4 \mu \mathrm{L}$ of water droplet was dripped on the samples for the measurement, with average results recorded upon averaging measurements at five different positions on the same sample. The diffuse reflectivity $(0.30-2.5 \mu \mathrm{m})$ was analyzed by an ultraviolet-visible-near-infrared (UV-Vis-NIR) 
spectrophotometer (Hitachi UH4150, Japan) with a barium sulfate $\left(\mathrm{BaSO}_{4}\right)$ integrating sphere. The mid-infrared spectral emissivity $(4-25 \mu \mathrm{m})$ at room temperature was measured by an FTIR spectrometer (Bruker Vertex 70, Germany) equipped with a goldcoated integrating sphere (PIKE America), via infrared measurement method.

Passive daytime radiative cooling evaluation: The passive daytime radiative cooling (PDRC) effect of prepared samples was evaluated under solar irradiation, simulated by xenon lamp (PLS-SXE300+, Perfectlight Technology Co., Ltd.). PDRC effect of these samples was associated with the temperature difference recorded by thermocouples under irradiation. Irradiation power was precisely controlled at $100 \mathrm{~mW} / \mathrm{cm}^{2}$ (one sun) with power meter, to adhere to the actual condition in reality.

The outdoor conditions during the test are: the on-site PDRC instrument with a solar intensity of $998 \mathrm{~W} / \mathrm{m}^{2}$ (Fuzhou, China, November 20, 2020), an ambient temperature of $37^{\circ} \mathrm{C}$, and a relative humidity of $82 \%$. In order to test the performance of PDRC under actual sunlight, we made a self-made closed environment test device, which is composed of a $5 \mathrm{~mm}$ thick glass plate and wrapped with aluminum foil. Heat insulation polystyrene foam is placed at the bottom of the box to reduce heat exchange between the inside and outside, and the thermocouple is placed between the polystyrene foam and the test sample. The top of the box is covered with a transparent PE film to reduce the potential impact of the external environment and internal heat convection of the self-made test box.

Durability test: The durability of synthesized samples was determined from their 
permeabilities, mechanical strengths and resilience against washing. In particular, the permeability of samples was measured with an automated air permeability instrument following the YG461E-11 test standard, while the universal mechanical testing machine (HD026S, HONGDA, China) was used to investigate the tensile strength of fabrics, with a clamp distance of $100.00 \mathrm{~mm}$ and tensile speed of $100.00 \mathrm{~mm} / \mathrm{min}$, following the standard test method GB/T3923.1-2013. Meanwhile, the PDRC performance of the samples was tested after washing (standard: AATCC 61). Such washing procedure was standardized across different samples through the application of laundering machine (HB 12P, NEWAVE LAB EQUIPMENTS CO., LTD) at $40{ }^{\circ} \mathrm{C}$, in the presence of 10 stainless steel balls. One washing cycle of $45 \mathrm{~min}$ is approximately equivalent to five times of commercial laundering.

\section{Results and discussion}

As shown in Scheme 1a, PDMS was firstly dispersed in water under ultrasonic stirring then the PTW, PDMS and curing agent were added to obtain hydrophobic finishing solution. The superhydrophobic surface was obtained after drying in an oven. 


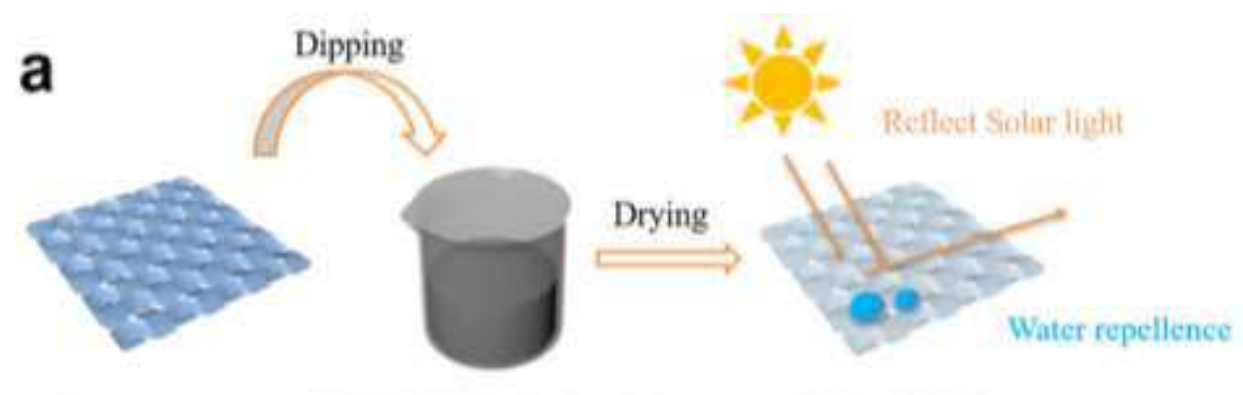

Bare cotton PDMS/PTW-mixed solution PDMS/PTW@ cotton Dolar energy
Absorbed $\left(q_{\text {men }}\right)$

Scheme 1. (a) The schematic diagram of PDMS/PTW@cotton. (b) Energy flows of radiative cooler, in which $q_{\text {sun }}$ is the absorbed solar radiation, $q_{\text {sky }}$ is the absorbed atmospheric radiation, $q_{\mathrm{rad}}$ is the thermal radiation, and $q_{\text {conv }+ \text { cond }}$ is the intrinsic cooling loss. (c) SEM images of PDMS/PTW@cotton.

To evaluate its PDRC effects, the modified cotton fabric was exposed to ambient under simulated solar irradiation as illustrated in Scheme 1b, whereby energy balance was performed to indicate the magnitude radiative cooling stream. According, the net radiative cooling power $\left(q_{\mathrm{cool}}\right)$ of the sample is the comprehensive embodiment of the Zhong SJ et al. 2021): Where $q_{\text {rad }}$ is denoted for energy radiated, $q_{\text {sun }}$ represents the energy absorbed simulated solar source, $q_{\text {sky }}$ is the atmospheric radiative energy absorbed, and $q_{\text {conv }+ \text { cond }}$ 
indicates the convective and conductive energies that associated to the heat transfer between skin, synthesized sample and air. Meanwhile, $T_{\mathrm{c}}$ and $T_{\mathrm{amb}}$ are the temperature of the radiative cooler and ambient air, respectively. In particular, the $\mathrm{q}_{\text {rad }}$ and $\mathrm{q}_{\text {sky }}$ can be determined from the following Eqs. (2)-(5).

$q_{\text {rad }}\left(T_{c}\right) \pi \int_{0}^{\infty} \int_{0}^{\frac{\pi}{2}} I_{B B}\left(T_{c}, \lambda\right) \varepsilon_{c}(\theta, \lambda) \sin (2 \theta) d \lambda d \theta$

$q_{\text {sky }}\left(T_{a m b}\right)=$

$2 \pi \int_{0}^{\infty} \int_{0}^{\frac{\pi}{2}} \cos \theta \sin \theta I_{B B}\left(\lambda, T_{a m b}\right) \varepsilon_{c}(\lambda, \theta) \varepsilon_{a m b}(\lambda, \theta) d \theta d \lambda$

$q_{\text {sun }}=$

$\int_{0}^{\infty} \varepsilon_{c}\left(\lambda, \theta_{\text {sun }}\right) I_{A M 1.5}(\lambda) d \lambda$

$q_{\mathrm{conv}+\mathrm{cond}}=$

$h A_{\mathrm{c}}\left(T_{a m b}-T_{\mathrm{c}}\right)$

$I_{\mathrm{BB}}\left(T_{\mathrm{c}}, \lambda\right)$ and $I_{\mathrm{BB}}\left(\lambda, T_{\mathrm{amb}}\right)$ in Eq. (2) and (3) are denoted for the spectral radiance of blackbody at temperature $T_{\mathrm{c}}$ and $T_{\mathrm{amb}}$, respectively, while $\varepsilon_{c}(\theta, \lambda)$ and $\varepsilon_{a m b}(\theta, \lambda)$ are the spectral and angular emittance of the radiative cooler and atmosphere. Whereas, $\theta_{\text {sun }}$ in Eq. (4) is assigned for the irradiation angle of the radiative cooler faces relative to simulated solar light and $I_{A M 1.5}(\lambda)$ being the AM $1.5 \mathrm{G}$ spectrum distribution of such radiation. As for Eq. (5), $h$ symbolizes the equivalent coefficient in consideration of both conductive and convective heat transfer, with $A_{\mathrm{c}}$ being the heat transfer area of 
the radiative cooler.

To achieve PDRC performance, the coating must satisfy a very stringent set of constraints that dictated by the energy balance in equation (4). It is necessary to

215 minimize $q_{\text {sun }}, q_{\text {sky }}$, and $q_{\text {conv+cond. }}$ While the designed coating must be spectrally 216 selective with strong emission between $8.0-13 \mu \mathrm{m}$, where the atmosphere window is transparent, and needs to have high reflectivity at Vis-NIR wavelengths.

PDMS/PTW cotton was fabricated by dipping method. The surface morphologies of PDMS/PTW cotton during fabrication are shown in Fig. 1. The pristine cotton surface is smooth with a groove structure (Fig. 1a). It can be seen in Fig. 1b that the rougher morphology. Closer magnification in Fig. 1c and Fig. 1d manifested the

223 intimate contact of needle-shaped PTW with the cotton fiber, attributed to the adhesive 224 effects arises from PDMS and the hydroxyl groups on cotton fiber. In order to prove the generality and practical value of our method, we modified polyester and nylon substrates. Fig. S1 is the SEM image of nylon and polyester modified by PDMS/PTW.

227 It can be seen that PTW is evenly covered on different types of substrates. It shows that 228 this method is suitable for the modification of different substrates and broadens its practical universality. 

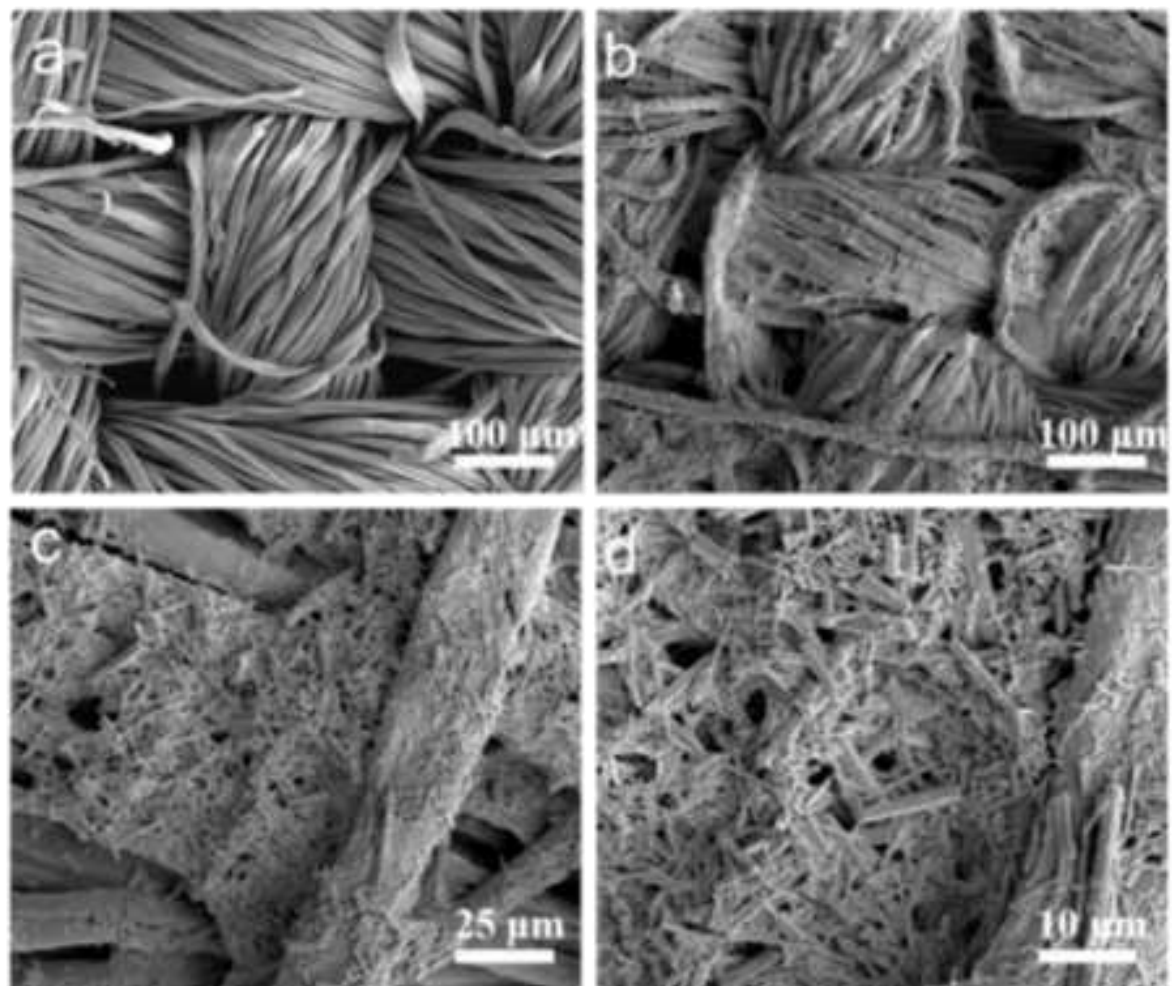

Figure 1. SEM images of pristine fabric (a) and PDMS/PTW@cotton at different magnification (b-d).

As indicated in Fig. 2a, the UV absorption of modified cotton fabric, in the range of 200-320 nm, is significantly higher than that of unmodified sample. Based on calculation, such improvement upon incorporation of PDMS/PTW could reach up to nearly $30 \%$ in the aforementioned UV range, implying better protection against solar irradiation. Meanwhile, the reflectivity of the prepared sample in the near infrared region could be served as an important indicator for its passive radiation cooling performance. The PDMS/PTW@cotton fabric realized with a high reflectivity of $83 \%$ in the range of $400-2500 \mathrm{~nm}$ (Fig. $2 \mathrm{~b}$ ), which associated to $\sim 25 \%$ improvement as compared to that of pristine cotton fabric. Such feature is essential for the daytime passive radiation cooling, as light in this mentioned region contributed to appreciable 
heating effects too. In addition, high infrared emissivity at the atmospheric window (8-

$24414 \mu \mathrm{m})$ are required too for prevailed PDRC. Further, we conducted near-infrared

245 reflectance and time-temperature tests on polyester and nylon samples. Fig. S2a and

246 Fig. S2c show that the reflectivity of nylon and polyester after modification has been

247 effectively improved. In Fig. S2b and Fig. S2d, it can be seen that the final temperature

248 of the modified sample is lower. The final temperature difference is mainly due to the

249 properties of the fabric itself. The content of PDMS/PTW coated on different fibers is

250 different and the degree of radiant cooling is different. Fig. $2 c$ and Fig. $2 d$ shows the

251 transmittance and emissivity of pristine and modified cotton fabric in mid-infrared

252 range, respectively, confirming the similarity of both samples in the context of mid-

253 infrared transmittance and emissivity. The combination of near-infrared reflectivity and

254 high mid-infrared emissivity endows limitless potential to the modified cotton fabric

255 for passive radiation cooling application. 

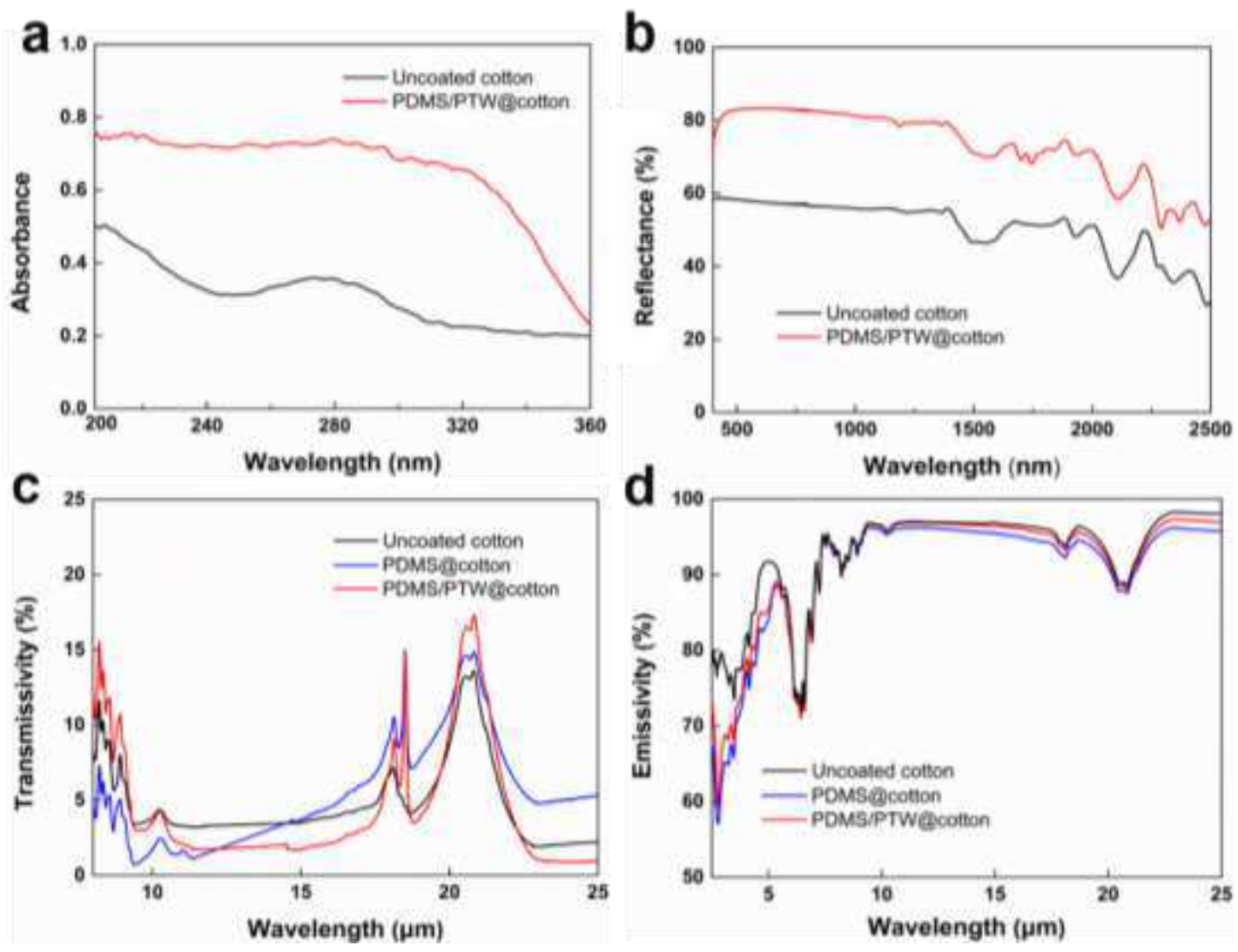

257 Figure 2. Spectrum of modified cotton fabric. (a) UV absorption curve (200-360 nm)

(b) Near-infrared reflectance curve $(0.4-2.5 \mu \mathrm{m})$. (c) Mid-infrared transmittance curve

(8-25 $\mu \mathrm{m})$. (d) Mid-infrared emissivity curve (4-25 $\mu \mathrm{m})$.

We compared the UV transmittance of the fabric before and after modification. $33.06 \%$ and $26.17 \%$ to $6.57 \%$ and $0.39 \%$ respectively in the range of UVA and UVB, which confirmed that the material has good UV resistance. The UPF value of modified cotton fabric reaches 122.79 , which is far greater than the UV protection UPF standard of 50+, reaching an effective protection level. It shows that this method can obtain good anti-ultraviolet performance when applied to cotton fabrics, and can effectively protect the human body from excessive ultraviolet radiation and damage the body. 
Table 1. UV protection parameters of uncoated cotton and PDMS/PTW@cotton.

\begin{tabular}{cccc}
\hline Sample & UPF & T(UVA/\%) & T(UVB/\%) \\
\hline Uncoated cotton & 4.21 & 33.06 & 26.17 \\
PDMS/PTW@cotton & 122.79 & 6.57 & 0.39 \\
\hline
\end{tabular}
with 2-5 $\mu \mathrm{m}$ PTW and 10-30 $\mu \mathrm{m}$ PTW before subjected to near infrared reflectance assessment under simulated solar light, as depicted in Fig. 3a. SEM images (Fig. 3b) indicated that the rod-like PTW $(10-30 \mu \mathrm{m})$ was deposited on the cotton fiber while a cross-linked structure was observed upon the deposition of small-sized PTW (right picture of Fig. 3b). The signals captured in NIR reflectance assessment (Fig. 3c) confirmed the greater reflectivity of 2-5 $\mu \mathrm{m}$ PTW-modified sample, thereby verifying its superior passive radiation cooling performance as opposed to that of 10-30 $\mu \mathrm{m}$ PTWmodified fabric. Meanwhile, a lower temperature increment rate was recorded by thermocouple underneath the irradiated cotton, with merely $31.0^{\circ} \mathrm{C}$ elevated from $25^{\circ} \mathrm{C}$ after 40 min of light exposure (Fig. 3d). On the other hand, fabric that modified with 10-30 $\mu \mathrm{m}$ PTW experienced greater temperature increment of $33.1^{\circ} \mathrm{C}$ under the same condition. From the perspective of particle size, particles of 2-5 $\mu \mathrm{m}$ have an advantage

282 in near-infrared reflection performance compared with larger particle sizes. This 283 phenomenon may be caused by incident radiation penetrating the sample particles and 284 being reflected by the grain boundaries. Produce diffuse reflection. As the particle size increases, the penetration depth plus the rate of grain boundary reduction decreases. At the same time, from the perspective of the preparation process, the larger particle size 
may not be able to pass the preparation process due to the weight, and the larger particle size may not be more effectively deposited on the cotton fiber through the PDMS as a binder due to the greater weight, which in turn causes performance loss.

In addition, we discussed PDRC samples prepared with different dipping times, as shown in Fig. 3e-f. Fig. 3e corresponds to the PDRC samples immersed in 1, 3, 5 times of PTW respectively. We can see that as the number of dipping increases, the PTW loaded on the fabric surface gradually increases. It was explicit that the temperature elevation decreased with impregnated layer, with PTW-5 layered-cotton exhibiting greatest cooling effects under simulated sun (Fig. 3f). Meanwhile, the effects of PTW concentration $(10-30 \mathrm{mg} / \mathrm{mL})$ towards the resultant near-infrared reflection of cotton were investigated too. Results in Fig. S3 indicated an increased NIR reflectivity as the precursor concentration increased from $10 \mathrm{mg} / \mathrm{mL}$ to $20 \mathrm{mg} / \mathrm{mL}$, confirming the role of PTW as effective NIR reflector. However, over-concentrated PTW precursor could prompt undesirable agglomeration, which worsen the dispersity of PTW on cotton in the modification step. This explained the reduced NIR reflectance reported in Fig. S3, which likely to yield to lower radiation cooling effects too. Surmising from these preliminary results, it is concluded that PTW in length of $2-5 \mu \mathrm{m}$ at $20 \mathrm{mg} / \mathrm{mL}$ served best for PDRC with five impregnated-layers. These optimum parameters were carried forward to the subsequent investigations. 

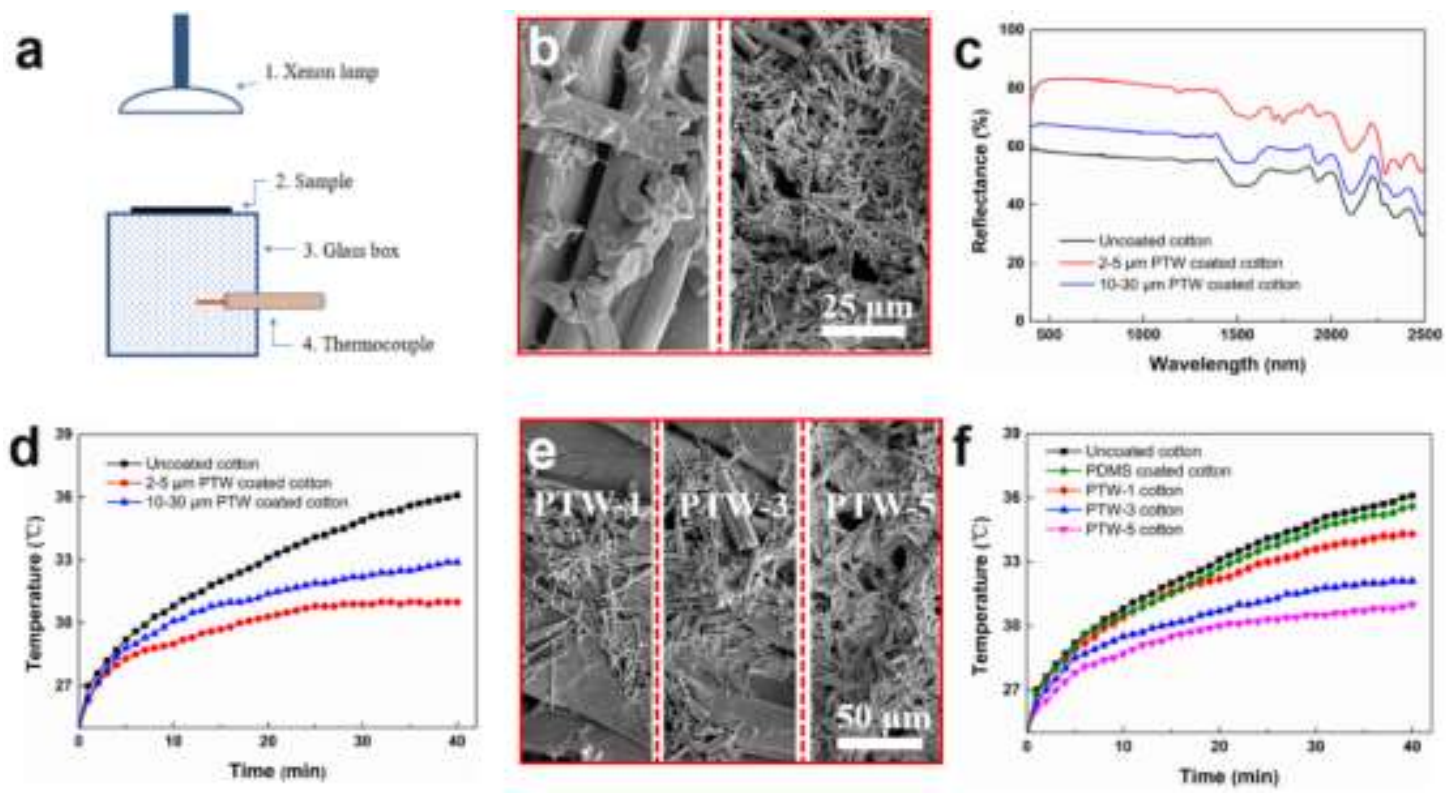

Figure 3. (a) Schematic diagram of simulated solar experimental device. (b) SEM images were impregnated for different times. (c) The near-infrared reflectance of different sizes of PTW. (d) Time-temperature curves for different sizes of PTW. (e)

SEM images of different sizes of PTW deposited on cotton fibers. (f)Time-temperature curves of the samples prepared with different impregnation times under simulated sunlight.

\section{PDRC performance test in sunlight}

In addition, the PDRC of samples through UV/NIR shielding was test under real sun by the setup illustrated in Fig. 4a. Fig. 4b is a cross-sectional view of a self-made cube test box. The device is composed of glass plates and wrapped with aluminum foil. Place the polystyrene foam at the bottom of the box, seal the top with PE film, and place the thermocouple between the polystyrene foam and the test sample. under the actual sunlight for a period of time, and the time-temperature curve of which 
sample put in the box and wrapped on the arm are as shown in Fig. 4c and Fig. 4f, respectively. PDMS/PTW@cotton showed an excellent PDRC performance that gives rise to $3.7^{\circ} \mathrm{C}-6.8^{\circ} \mathrm{C}$ temperature reduction, as compared to that of unmodified cotton fabric, under direct sunlight irradiation (Fig. 4c). Significantly, an average reduction of $\sim 5.1{ }^{\circ} \mathrm{C}$ was recorded within $11.30 \mathrm{am}-12.30 \mathrm{pm}$ by the modified cotton fabric in current study. The time-temperature curve obtained in Fig. $4 \mathrm{f}$ shows that compared with unmodified cotton fabric, modified cotton fabric has a relatively lower temperature curve, and the temperature difference fluctuates between $3.1{ }^{\circ} \mathrm{C}$ and $4.7{ }^{\circ} \mathrm{C}$. The average temperature difference It is $4.2^{\circ} \mathrm{C}$. These data show that the modified cotton fabric can also exert good PDRC performance in actual use. Such inspiring result can be attributed to the significantly increased NIR reflectance of PDMS/PTW@cotton compared with bare cotton, causing plummeted absorption of external solar heat. Comfort and breathability are very important for the wearer. The air permeability of cotton fabric before and after finishing is shown in Fig. S4. After modification, the air permeability of all fabrics decreased slightly, indicating the success adherence of coating layer onto the cotton fiber. Specifically, the permeability of PDMS/DI-treated samples decreased $2.7 \%$ while PDMS/PTW/DI-treated fabric marked higher reduction of $24.6 \%$. The addition of PTW has a certain negative effect on the air permeability of cotton fabric, but it still maintains a certain air permeability. 

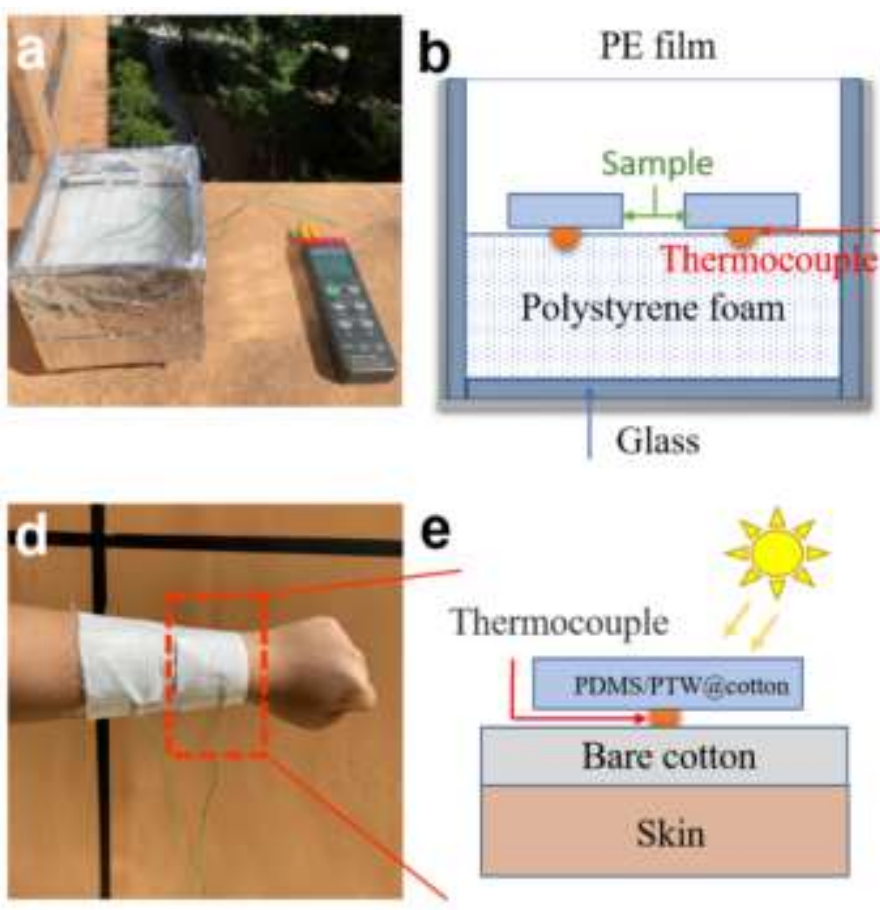

Thermocouple

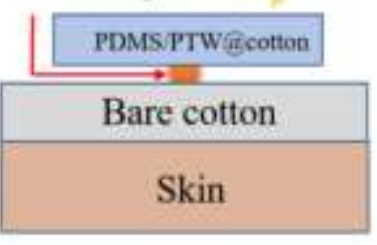

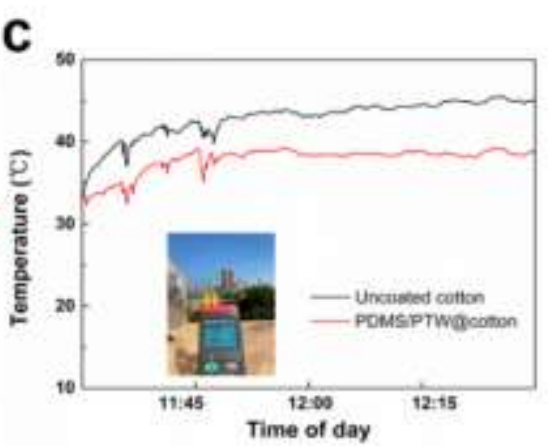

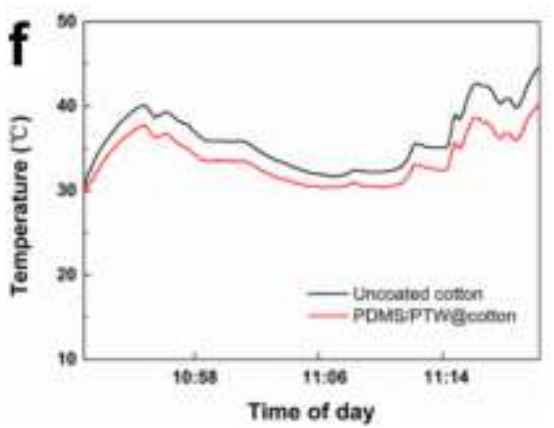

Figure 4. PDRC performance test of the sample under actual sunlight irradiation. (a)

Self-made test box and thermocouple thermometer photos. (b) Self-made test box diagram of section. (c) The time-temperature curve of the thermocouple thermometer under the actual sunlight irradiation is obtained by self-made test box. (d) Photos of modified fabric actually worn and connected thermocouple thermometers in sunlight. (e) The cross-section diagram of wearing method and thermocouple placement position. (f) The time-temperature curve of the thermocouple thermometer under the actual wearing condition.

\section{Self-cleaning properties of modified cotton fabrics}

PTW was evenly distributed on the cotton fiber with the assistance of water solvent, while its intimate bounding with base fabric was conferred by the hydrogen bonding established by PDMS and cotton fiber. As such, a low surface energy with high roughness was established upon PDMS/PTW modification, endowing the cotton 
superhydrophobic feature and self-cleaning ability. Evidently, both bare cotton and PDMS/PTW@cotton were put into methylene blue solution (MB), with only the former being wetted and stained (Fig. 5a).PDMS/PTW@cotton, on the other hand, retained its original clean surface with no blueish marks observed. In addition to MB, several common drinks, such as tea, coffee, cola, were selected as modeled dirt for staining test due to their recalcitrance against cleaning. It was found that all the three dirt droplets

360 remained spherically on the surface of the fabric without penetrating it (Fig. 5b), which demonstrated the excellent protection against these potential staining agents in daily life. In addition, it can be seen that the CA of PDMS/PTW@cotton was still above $150^{\circ}$ after keeping at room temperature for about 6 months (Fig. S5), indicating the excellent stability of the superhydrophobicity against ambient degradation.

Additionally, it is note that the PDRC performance of fabric can be affected due to the surface accumulation of dust and other similar pollutants. Evidently, coffee powder-accumulated sample (before cleaning) exhibited slight higher temperature ramping rate as compared to the clean sample under sunlight irradiation, with $0.8^{\circ} \mathrm{C}$ difference recorded after $40 \mathrm{~min}$. This indicated the adverse impact of ash layer on PDRC ability of the cotton cloth, thereby justified the importance of self-cleaning for sustained cooling. 

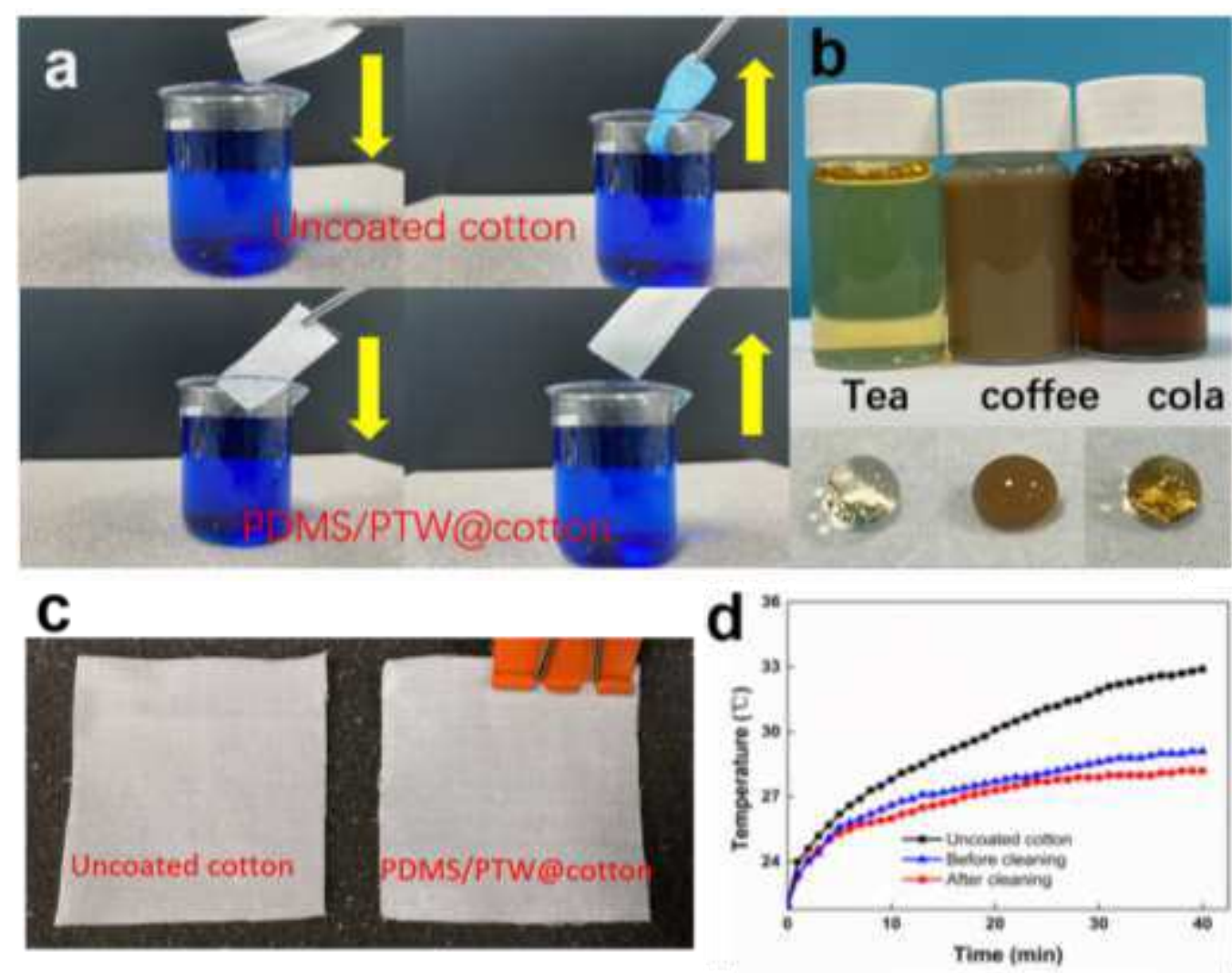

Figure 5. (a) Different performance of modified and unmodified cotton fabric in methylene blue dyed water. (b) Difficult infiltration of modified cotton fabric by common liquids in daily life. (c) Optical photos of modified cotton fabric before and after modification. (d) Time-temperature curve of modified cotton fabric under simulated sunlight before and after self-cleaning (coffee powder was dispersed as modelled ash layer).

\section{Mechanical properties of modified cotton fabric}

The resilience of PDMS/PTW@cotton against mechanical disturbances is another important factor for effective PDRC. In this context, ultrasonic cleaning at $40 \mathrm{KHz}$ was employed to evaluate the durability of the synthesized sample. As shown in Fig. 6a, the contact angle of sample remained high at $\sim 150{ }^{\circ}$ for 30 min of ultrasonicated., and 
also showed good stability (Fig. S6), and the two modified sample showed good hydrophobic performance after $30 \mathrm{~min}$ ultrasonic treatment. This confirms the stability of the prepared samples under ultrasonic conditions, and also illustrates the good adhesion of the superhydrophobic coating to different substrates.

Similar resilience and durability were also confirmed in normal washing too, judging from the nearly invariant CA even after 20 cycles of washing (Fig. 6b). Moreover, the influence of different washing times on PDRC performance was illustrated in Fig. 6c, in the form of time-temperature curves. Apparently, the PDRC performance of the samples was gradually decreased, thereby yielding to the elevating temperature profiles in conjecture with increased washing cycles. Such observable can be attributed to the peeling off of the PTW/PDMS coating, resulted from the repeated washing cycle.

To investigate the influence of the coating on physical strength, the mechanical properties of pristine cotton and PDMS/PTW@cotton were tested via a universal mechanical testing machine. As shown in Fig. $6 \mathrm{~d}$ and Table S1, the mechanical strength of modified cotton only decreased by $4.30 \%$ in warp direction and $19.04 \%$ in weft direction. This phenomenon may be caused by the multiple heat treatment in the preparation process the embedment of PTW that frictionally tapers the yarn of the fabric. However, sufficient mechanical strength was retained after the modification, indicating the potential application in daily life. 

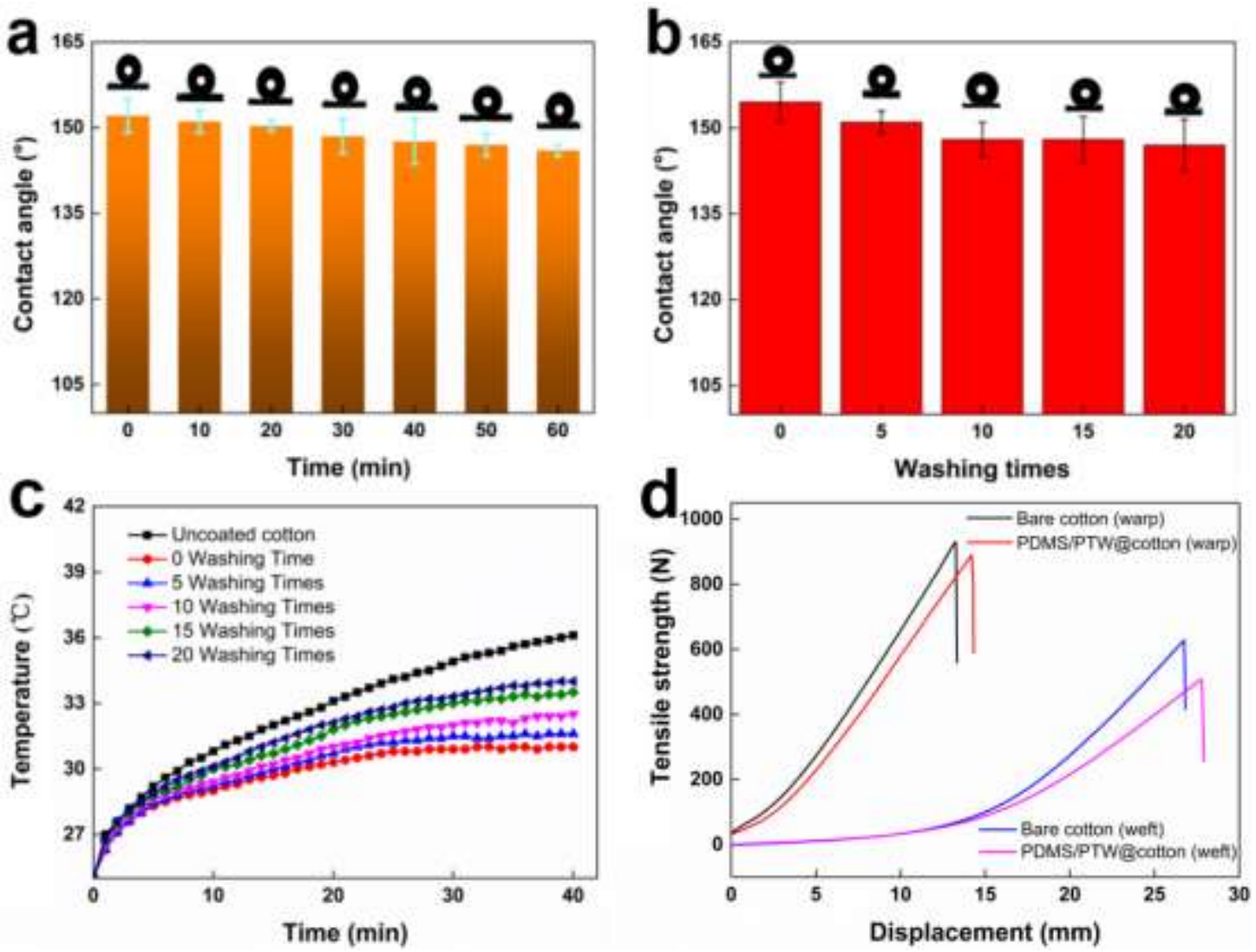

Figure 6. (a) Contact Angle of cotton fabric after different ultrasonic time and

corresponding droplet images. (b) Contact Angle and corresponding droplet image of

cotton fabric after four intensification washes. (c) Tensile strength of pristine fabric and modified fabric. (d) Time-temperature curves of cotton fabrics with different washing times under simulated sunlight.

Meanwhile, the hydrophobicity of the fabricated sample can also be tuned facilely with 30 s oxygen plasma treatment. As indicated in Fig. 7a, the CA of sample can be

413 dramatically reduced to $0^{\circ}$, from its initial $>150^{\circ}$, after the aforesaid treatment, while regaining back its superhydrophobicity after $30 \mathrm{~min}$ of heat treatment at $90{ }^{\circ} \mathrm{C}$. Such superhydrophobic-superhydrophilic transition was repeated for 5 cycles with no traceable loss observed, judging from the invariant $\mathrm{CA}$ at both ends (Fig. 7a). 
417 Fundamentally, the superhydrophobic-superhydrophilic transition was controlled by 418 the surface functional groups, whereby the oxygen plasma treatment prompted the 419 anchoring of hydrophilic groups on the cotton fiber surface. The subsequent heating restore back its original superhydrophobicity. The scheme of the conversion is illustrated in Fig. 7b.

423
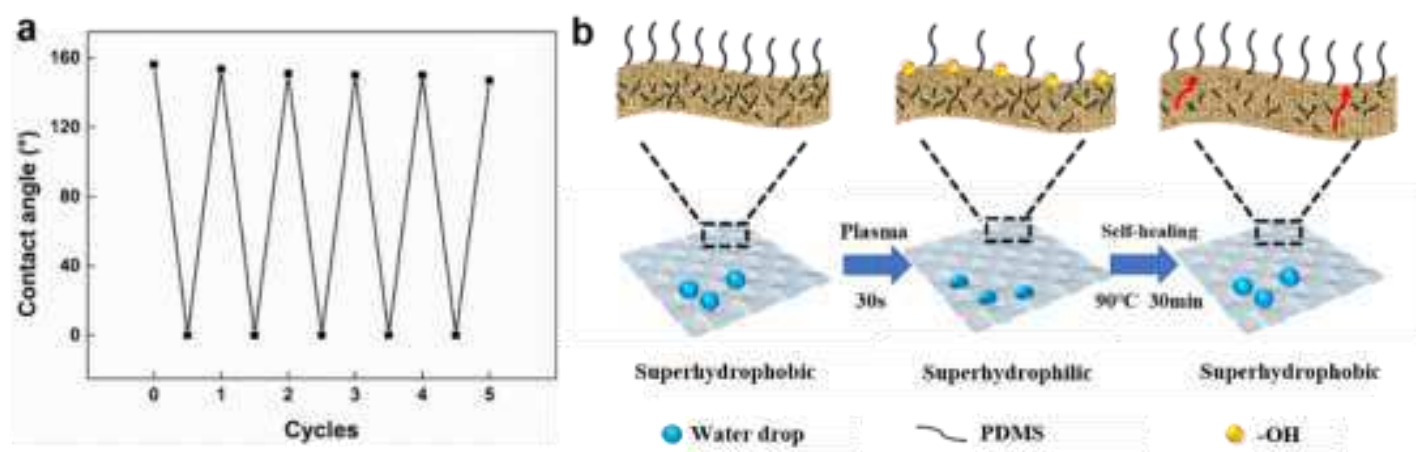

Figure 7. (a) Contact angle of the superhydrophobic cotton fabric after plasma etching and heating treatment for five cycles. (b) Schematic illustrations of the plasma/selfhealing mechanism.

\section{Conclusion}

In this paper, we prepared super hydrophobic PDMS/PTW coated cotton fabrics through a simple dip-coating protocol and tested their performance in passive daytime radiation cooling (PDRC). Results confirmed that the coating layer with smaller PTW particles $(2-5 \mu \mathrm{m})$ promotes PDRC of cotton with its enhanced reflectivity of $83 \%$ in the wavelength of $400-2500 \mathrm{~nm}$, which is about $25 \%$ higher than that of the bare cotton. Concurrently, the emissivity of the modified cotton was improved to $90 \%$ in the atmospheric window, which contributed to enhanced PDRC too. As results, the average 
temperatures of modified fabric recorded are $5.1{ }^{\circ} \mathrm{C}$ and $3.8^{\circ} \mathrm{C}$ lower than that of the original fabric, for PDRC assessment under direct sunlight and in human test, respectively. These results verified the adequacy of the proposed dip-coating method, as well as the competency of PDMS/PTW coating layer in improving PDRC of cotton fabric. In addition, the resultant low surface energy after PDMS/PTW deposition further imparted superhydrophobicity, and hence self-cleaning feature to the coated sample, which is important for a sustained PDRC against dirt-suppression. At the same time, due to the strong hydrogen bond between PDMS and cotton fiber, the modified fabric also has good mechanical properties and washing resistance. In conclusion, durable PDRC fabric with self-cleaning attribute was successfully developed herein, under a simple and environmentally friendly dip-coating method, which makes it potentially useful for summer outdoor outerwear and other applications.

\section{Acknowledgements}

The authors thank the National Natural Science Foundation of China (51972063, 51502185), Natural Science Foundation of Fujian Province (2019J01256), 111 Project (No. D17005), China postdoctoral science foundation (Pre-station, No. 2019TQ0061).

\section{Reference:}

Barros SC, Silva MM (2018) Seeking the lowest phase transition temperature in a cellulosic system for textile applications. Cellulose 25: 3163-3178. 
Cai LL, Song AY, Li W, Hsu PC, Lin DC, Catrysse PB, Liu YY, Peng YC, Chen J, Wang HX, Xu JW, Yang AK, Fan SH, Cui Y (2018) Spectrally Selective Nanocomposite Textile for Outdoor Personal Cooling. Adv Mater 30: 1802152.

Spector JT, Bonauto DK, Sheppard L, Busch-Isaksen T, Calkins M, Adams D, Lieblich M, Fenske RA (2016) A Case-Crossover Study of Heat Exposure and Injury Risk in Outdoor Agricultural Workers. PLoS One 11: e0164498.

Kjellstrom T, Briggs D, Freyberg C, Lemke B, Otto M, Hyatt O (2016) Heat, Human Performance, and Occupational Health: A Key Issue for the Assessment of Global Climate Change Impacts. Annu Rev Public Health 37: 97-112.

Lian YL, Yu H, Wang MY, Yang XN, Li Z, Yang F, Wang Y, Tai HL, Liao YL, Wu JY, Wang XR, Jiang YD, Tao GM (2020) A multifunctional wearable E-textile via integrated nanowire-coated fabrics. J Mater Chem C 8: 8399-8409.

Miao DG, Jiang SX, Liu J, Ning X, Shang SM, Xu JT (2017) Fabrication of copper and titanium coated textiles for sunlight management. J Mater Sci Mater Electron 28: 9852-9858.

Panwar K, Jassal M, Agrawal AK (2017) $\mathrm{TiO}_{2}-\mathrm{SiO}_{2}$ Janus particles treated cotton fabric for thermal regulation. Surf Coat Technol 309: 897-903.

Peng LH, Su B, Yu AB, Jiang XC (2019) Review of clothing for thermal management with advanced materials. Cellulose 26: 6415-6448.

Song YN, Ma RJ, Xu, L, Huang HD, Yan DX, Xu JZ, Zhong GJ, Lei J, Li ZM (2018) Wearable Polyethylene/Polyamide Composite Fabric for Passive Human Body Cooling. ACS Appl Mater Interfaces 10: 41637-41644.

Sun KY, Dong HS, Kou Y, Yang HN, Liu HQ, Li YG, Shi Q (2021) Flexible graphene aerogel-based phase change film for solar-thermal energy conversion and storage in personal thermal management applications. Chem Eng J 419: 129637.

Yang YX, Bao XM, Wang Q, Wang P, Zhou M, Yu YY (2021) Thermo-responsive 
cotton fabric prepared by enzyme-initiated "graft from" polymerization for moisture/thermal management. Cellulose 28: 1795-1808.

Yu X, Li Y, Yin X, Wang XF, Han YH, Si Y, Yu JY, Ding B (2019) Corncoblike, Superhydrophobic, and Phase-Changeable Nanofibers for Intelligent Thermoregulating and Water-Repellent Fabrics. ACS Appl Mater Interfaces 11: 39324-39333.

Lu Y, Xiao XD, Fu J, Huan CM, Qi S, Zhan YJ, Zhu YQ, Xu G (2019) Novel smart textile with phase change materials encapsulated core-sheath structure fabricated by coaxial electrospinning. Chem Eng J 355: 532-539.

Peng LH, Chen WF, Su B, Yu AB, Jiang XC (2019) $\mathrm{CsxWO}_{3}$ nanosheet-coated cotton fabric with multiple functions: UV/NIR shielding and full-spectrum-responsive self-cleaning. Appl Surf Sci 475: 325-333.

Wong A, Daoud WA, Liang HH, Szeto YS (2015) Application of rutile and anatase onto cotton fabric and their effect on the NIR reflection/surface temperature of the fabric. Sol Energy Mater Sol Cells 134: 425-437.

Yang M, Zou WZ, Guo J, Qian ZC, Luo H, Yang SJ, Zhao N, Pattelli L, Xu J, Wiersma DS (2020) Bioinspired "Skin" with Cooperative Thermo-Optical Effect for Daytime Radiative Cooling. ACS Appl Mater Interfaces 12: 25286-25293.

Yuan H, Li T, Wang Y, Ma PM, Du ML, Liu TX, Yan YY, Bai HY, Chen MQ, Dong WF (2020) Photoprotective and multifunctional polymer film with excellent nearinfrared and UV shielding properties. Compos Commun 22: 100443.

Zhou Y, Song HM, Liang JW, Singer M, Zhou M, Stegenburgs E, Zhang N, Xu C, Ng T, Yu ZF, Ooi B, Gan QQ (2019) A polydimethylsiloxane-coated metal structure for all-day radiative cooling. Nat Sustain 2: 718-724.

Hsu PC, Song AY, Catrysse PB, Liu C, Peng YC, Xie J, Fan SH, Cui Y (2016) Radiative human body cooling by nanoporous polyethylene textile. Science 353: 1019-1023. 
Wu K, Yu LP, Lei CX, Huang JX, Liu DY, Liu Y, Xie YS, Chen F, Fu Q (2019) Green Production of Regenerated Cellulose/Boron Nitride Nanosheet Textiles for Static and Dynamic Personal Cooling. ACS Appl Mater Interfaces 11: 40685-40693.

Catrysse PB, Song AY, Fan SH (2016) Photonic Structure Textile Design for Localized Thermal Cooling Based on a Fiber Blending Scheme. ACS Photonics 3: 24202426.

Fan WJ, He Q, Meng KY, Tan XL, Zhou ZH, Zhang GQ, Yang J, Wang ZL (2020) Machine-knitted washable sensor array textile for precise epidermal physiological signal monitoring. Sci Adv 6: eaay2840.

Raman AP, Anoma MA, Zhu LX, Rephaeli E, Fan SH (2014) Passive radiative cooling below ambient air temperature under direct sunlight. Nature 515: 540-4.

Zhang HW, Ly KCS, Liu XH, Chen ZH, Yan M, Wu ZL, Wang X, Zheng YB, Zhou H, Fan TX (2020) Biologically inspired flexible photonic films for efficient passive radiative cooling. P Natl Acad Sci USA 117: 14657-14666.

Zhai Y, Ma YG, David SN, Zhao DL, Lou RN, Tan G, Yang RG, Yin XB (2017) Scalable-manufactured randomized glass-polymer hybrid metamaterial for daytime radiative cooling. Science 355: 1062-1066.

Qi YL, Xiang B, Tan WB, Zhang J (2017) Hydrophobic surface modification of $\mathrm{TiO}_{2}$ nanoparticles for production of acrylonitrile-styrene-acrylate terpolymer $/ \mathrm{TiO}$ composited cool materials. Appl Surf Sci 419: 213-223.

Shi NN, Tsai CC, Camino F, Bernard GD, Yu NF, Wehner R (2015) Keeping cool Enhanced optical reflection and radiative heat dissipation in Saharan silver ants. Science 349: 298-301.

Sala-Casanovas M, Krishna A, Yu ZQ, Lee, J (2019) Bio-Inspired Stretchable Selective Emitters Based on Corrugated Nickel for Personal Thermal Management. Nanosc Microsc Therm 23: 173-187. 
Huang WL, Chen YJ, Luo Y, Mandal J, Li WX, Chen MJ, Tsai CC, Shan ZQ, Yu NF, Yang Y (2021) Scalable Aqueous Processing-Based Passive Daytime Radiative Cooling Coatings. Adv Funct Mater 31: 2010334.

Anand J, Sailor DJ, Baniassadi A (2021) The relative role of solar reflectance and thermal emittance for passive daytime radiative cooling technologies applied to rooftops. Sustain Cities Soc 65: 102612.

Dong SM, Quek JY, Van Herk AM, Jana S (2020) Polymer-Encapsulated $\mathrm{TiO}_{2}$ for the Improvement of NIR Reflectance and Total Solar Reflectance of Cool Coatings. Ind Eng Chem Res 59: 17901-17910.

Gu B, Liang KF, Zhang T, Qiu FX, Yang DY, Chen MM (2020) Multifunctional laminated membranes with adjustable infrared radiation for personal thermal management applications. Cellulose 27: 8471-8483.

Huang X, Liu DF, Li N, Wang JF, Zhang ZJ, Zhong MF (2020) Single novel $\mathrm{Ca}_{0.5} \mathrm{Mg}_{10.5}\left(\mathrm{HPO}_{3}\right)_{8}(\mathrm{OH})_{3} \mathrm{~F}_{3}$ coating for efficient passive cooling in the natural environment. Solar Energy 202: 164-170.

Sabzi D, Haseli P, Jafarian M, Karimi G, Taheri M (2015) Investigation of cooling load reduction in buildings by passive cooling options applied on roof. Energy Build 109: $135-142$.

Song YN, Lei MQ, Lei J, Li ZM (2020) A Scalable Hybrid Fiber and Its Textile with Pore and Wrinkle Structures for Passive Personal Cooling. Adv Mater Technol 5: 2000287.

Zhong SJ, Yi LM, Zhang JW, Xu TQ, Xu L, Zhang X, Zuo T, Cai Y (2021) Self-cleaning and spectrally selective coating on cotton fabric for passive daytime radiative cooling. Chem Eng J 407: 127104. 
Corresponding author email: jyhuang@fzu.edu.cn shielding

Taipei City 24301, Taiwan

Nanyang Avenue, Singapore PDMS/PTW@cotton fabric for efficient UV/NIR light

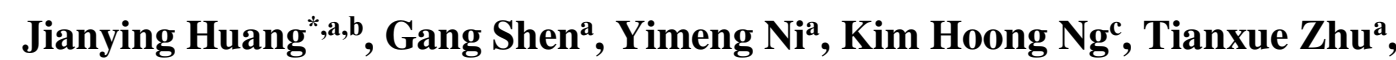 \\ Shuhui Li ${ }^{\mathrm{a}}$, Xiao Li ${ }^{\mathrm{a}, \mathrm{b}}$, Weilong Cai ${ }^{\mathrm{a}, \mathrm{b}}$, Zhong Chen ${ }^{\mathrm{d}}$
}

${ }^{a}$ College of Chemical Engineering, Fuzhou University, Fuzhou 350116, P. R. China

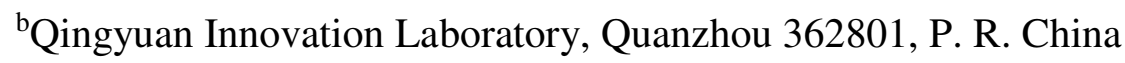

${ }^{c}$ Department of Chemical Engineering, Ming Chi University of Technology, New

${ }^{\mathrm{d}}$ School of Materials Science and Engineering, Nanyang Technological University, 50 


\section{List of Table \& Figure Caption:}

574 Table S1. Mechanical properties of uncoated cotton and modified cotton.

575 Figure S1. SEM images of PDMS/PTW modified polyester (a) and nylon (b).

576 Figure S2 (a-b) Near-infrared reflectance of modified polyester fabric and time577 temperature curves under simulated sunlight. (c-d) Near-infrared reflectance of 578 modified nylon fabric and time-temperature curves under simulated sunlight.

579 Figure S3. Infrared reflectance of cotton cloth prepared with different concentrations 580 of PTW.

581 Figure S4. Air permeability of common cotton and fabric prepared with two different 582 solutions (PDMS coated cotton without PTW).

583 Figure S5. Contact Angle of PDMS/PTW modified cotton cloth after long time 584 placement.

585 Figure S6 The contact Angle of nylon and polyester modified by PDMS/PTW at 586 different ultrasonic time. 
588 Table S1. Mechanical properties of uncoated cotton and modified cotton.

\begin{tabular}{llcc}
\hline & $\begin{array}{c}\text { Tensile force at } \\
\text { breakage }(\mathbf{N})\end{array}$ & $\begin{array}{c}\text { Elongation at break } \\
(\boldsymbol{\%})\end{array}$ & $\begin{array}{c}\text { Strength reduction } \\
(\boldsymbol{\%})\end{array}$ \\
\hline Uncoated cotton & 929.85 (warp) & 13.26 (warp) & 0 (warp) \\
& 628.18 (weft) & 26.76 (weft) & 0 (weft) \\
Modified cotton & 889.89 (warp) & 14.19 (warp) & 4.30 (warp) \\
& 508.58 (weft) & 27.76 (weft) & 19.04 (weft) \\
\hline
\end{tabular}

589

590
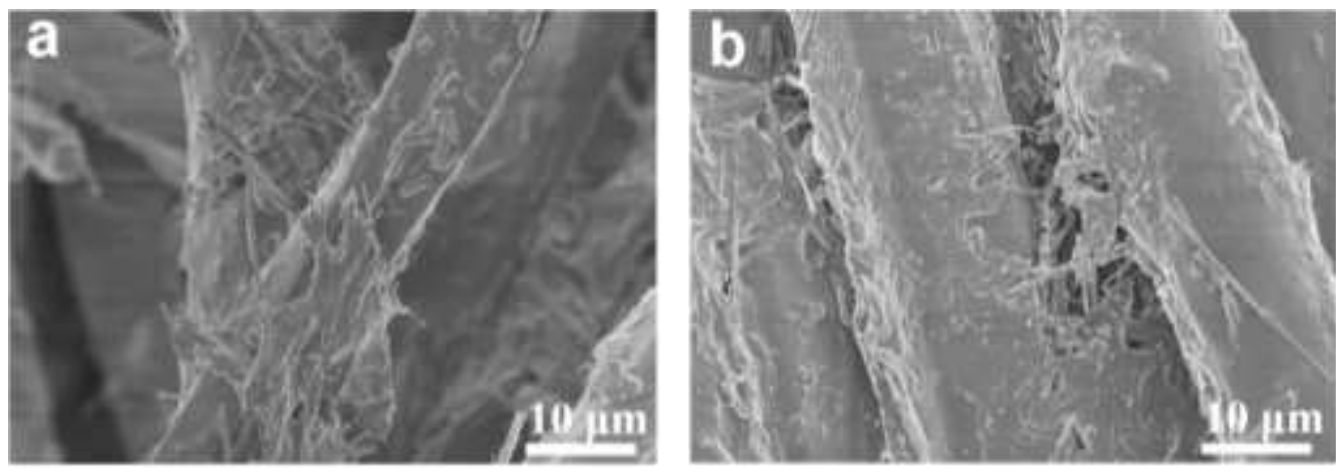

591 Figure S1. SEM images of PDMS/PTW modified polyester (a) and nylon (b). 

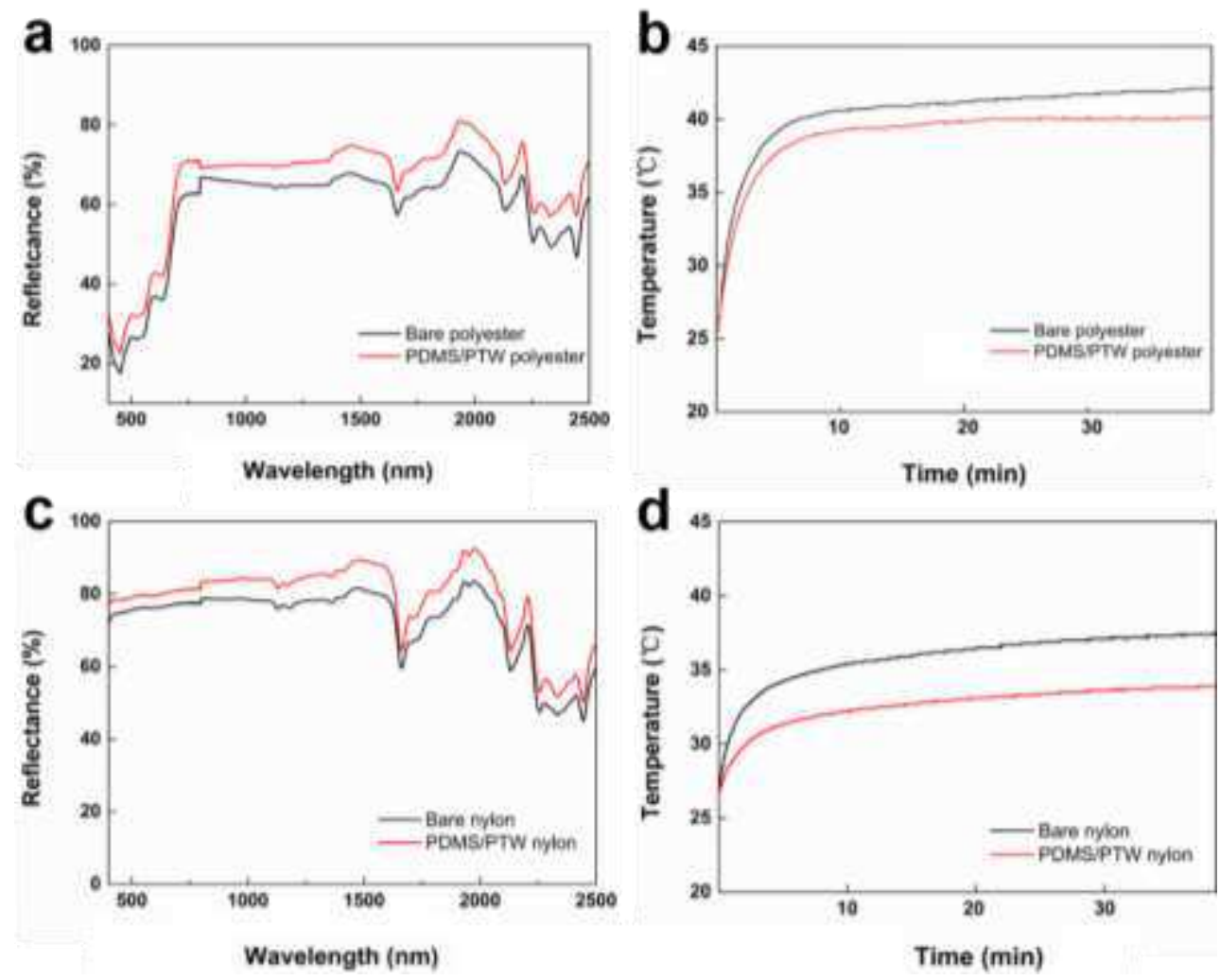

594 Figure S2 (a-b) Near-infrared reflectance of modified polyester fabric and timetemperature curves under simulated sunlight. (c-d) Near-infrared reflectance of modified nylon fabric and time-temperature curves under simulated sunlight.

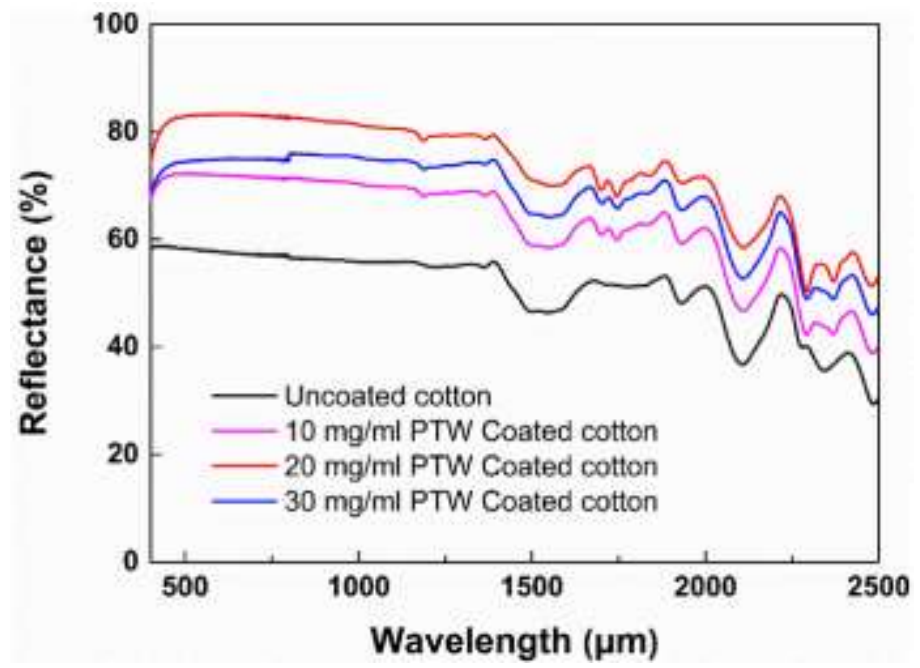

599 Figure S3. Infrared reflectance of cotton cloth prepared with different concentrations 600 of PTW. 


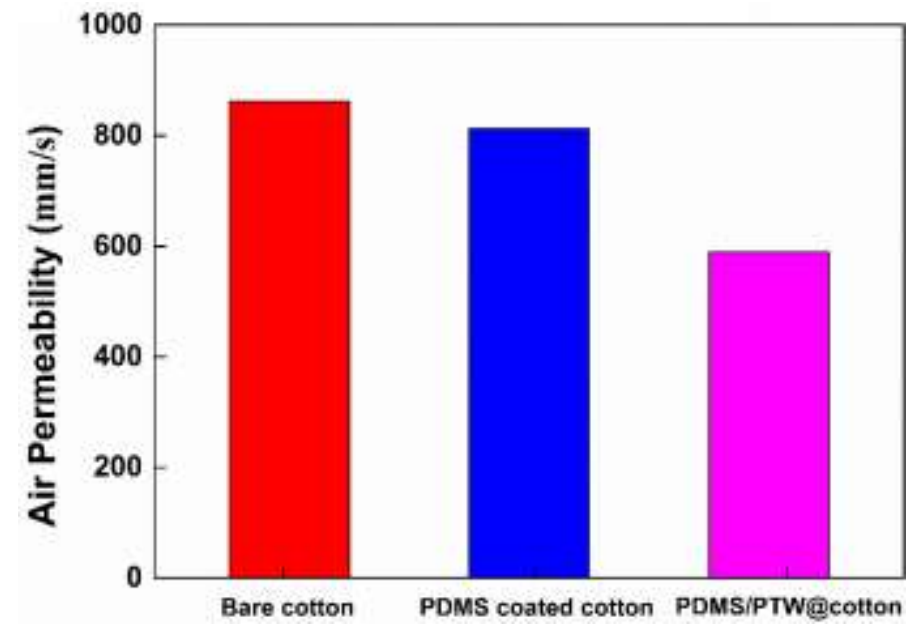

603 Figure S4. Air permeability of common cotton and fabric prepared with two different 604 solutions (PDMS coated cotton without PTW).

605

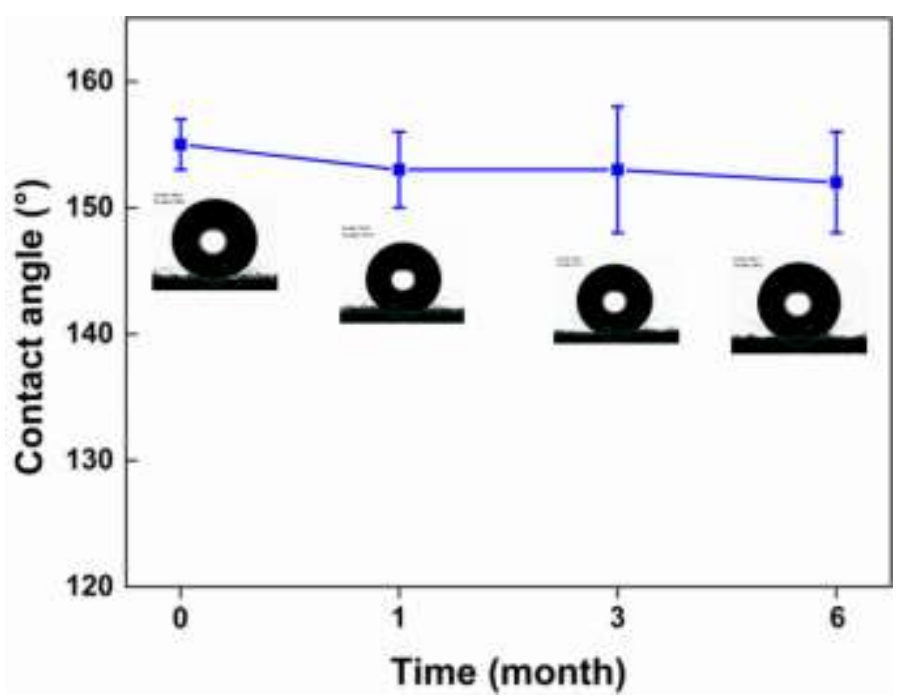

Figure S5. Contact Angle of PDMS/PTW modified cotton cloth after long time 608 placement. 


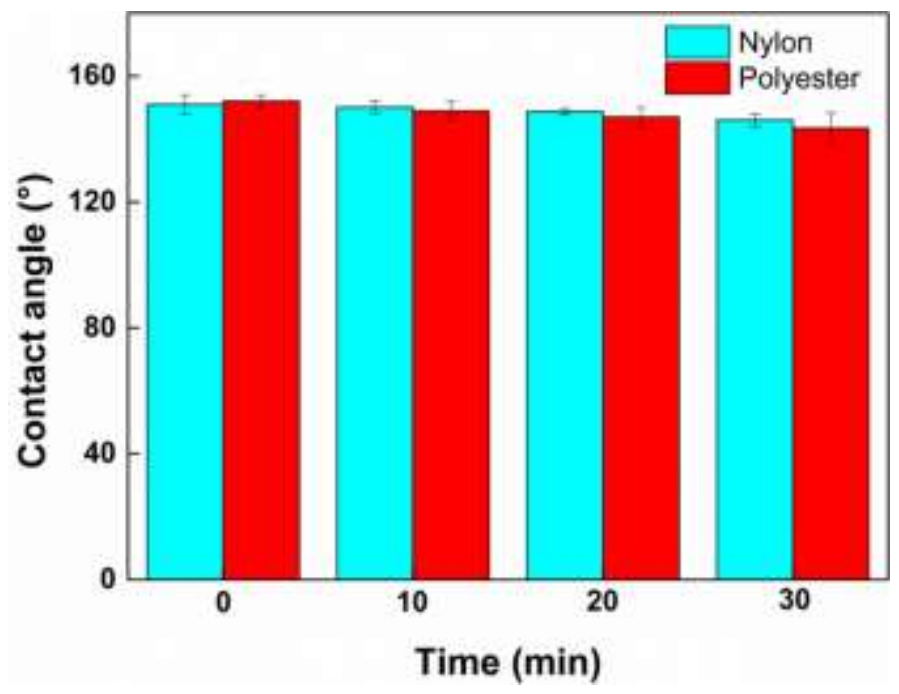

611 Figure S6. The contact Angle of nylon and polyester modified by PDMS/PTW at 612 different ultrasonic time.

613

614 